Meta

Journal des traducteurs

Translators' Journal

\title{
Réflexions sur un numéro spécial
}

\section{Brigitte Lépinette}

Volume 38, numéro 4, décembre 1993

Le Je du traducteur

The $I$ of the Translator

URI : https://id.erudit.org/iderudit/003469ar

DOI : https://doi.org/10.7202/003469ar

Aller au sommaire du numéro

Éditeur(s)

Les Presses de l'Université de Montréal

ISSN

0026-0452 (imprimé)

Découvrir la revue

Citer cet article

Lépinette, B. (1993). Réflexions sur un numéro spécial. Meta, 38(4), 597-602. https://doi.org/10.7202/003469ar d'utilisation que vous pouvez consulter en ligne.

https://apropos.erudit.org/fr/usagers/politique-dutilisation/ 


\title{
RÉFLEXIONS SUR UN NUMÉRO SPÉCIAL
}

\author{
BRIGITTE LÉPINETTE \\ Université de Valence, Valence, Espagne
}

Cette partie de ce numéro était conçue au départ comme une exploration du monde des traducteurs professionnels espagnols, lesquels se seraient «confessés» au profit des lecteurs de la revue, en retraçant l'itinéraire qui les avait conduits - volontairement ou non - à cette activité, en évoquant des anecdotes et les difficultés du métier propres à leur pays, mais aussi en analysant la pratique qui constitue leur travail au quotidien, dans un «ici et maintenant». Nous aurions de cette manière constitué une sorte de «dossier» sur ce que ce domaine peut avoir de spécifique, dans cette partie de la péninsule ibérique. Il nous semblait, a priori, qu'un pays comme l'Espagne, qui publie un très grand nombre de traductions littéraires mais aussi techniques, avait beaucoup à dire sur le sujet. J'ai ainsi frappé à de nombreuses portes pour expliquer le projet. Cependant il ne soulevait pas d'enthousiasme particulier. Les traducteurs ne se sont pas précipités devant leur machine à écrire pour remplir fébrilement leur feuille blanche, profitant de l'occasion qui leur était offerte, de pouvoir parler d'eux-mêmes, de leur vie et de leur métier. Il a fallu se rendre à l'évidence: les traducteurs professionnels espagnols n'ont pas particulièrement envie d' «écrire leurs mémoires»; leur profession leur apparaît surtout un métier qu'ils ont exercé (ou qu'ils exercent) - tout prosaïquement et comme tout un chacun - pour pouvoir vivre. Ils sont apparemment de l'avis que sur le plan personnel, ils ont peu à dire sur le sujet. J'ajouterai que ce manque d'intérêt dans la «peinture de soi-même» correspond peut-être aussi à une tendance générale observée chez les écrivains espagnols. Dans ce pays, l'autobiographie est un genre littéraire assez peu cultivé et ne bénéficie pas de la tradition qu'il peut avoir en France, par exemple. Il n'y a pas ici à rechercher les causes du phénomène, mais seulement à en prendre acte.

Sur un autre plan, ce silence des traducteurs professionnels peut aussi laisser supposer qu'ils considèrent qu'en la matière il n'y a pas à généraliser à partir des textes qu'ils ont traduits, encore moins à théoriser. L'artisan qui donne forme à la matière qu'il travaille le jardinier qui taille son arbre ou le sculpteur devant son bloc de marbre — ne pense pas non plus avoir à faire l'inventaire de ses gestes ou la description de la pièce achevée. Le résultat seul importe et, pour cela, il n'y a qu'à regarder. Chacun doit apprendre à guider son propre bras.

L'activité d'un traducteur serait donc, dans cette optique, indépendante des savoirs en linguistique constrastive ou en théorie de la traduction. Tous ces «métadiscours» ne seraient à la traduction que ce qu'une analyse grammaticale d'une langue est à n'importe quel texte écrit, et il y a longtemps qu'on a déjà dit qu'il est impossible d'écrire une phrase correcte, sans savoir en analyser les différentes composantes. Bref, on peut faire de la prose sans en connaître la nature. Pour les traducteurs, il en irait de même. Tout serait affaire d'entraînement et de pratique.

Enfin, de cette absence de désir de parler que nous avons constatée, il n'est pas interdit de déduire que, pour ces praticiens, la traduction constitue un domaine complètement indépendant de celui de la création. Elle n'est pas conçue comme une propédeutique qui, de la reformulation des textes d'autrui dans un premier temps, permettrait de passer, dans un second, à la création du sien propre. Le «si vous traduisez toujours, on ne vous traduira 
jamais» leur apparaîtrait ainsi comme l'aphorisme énoncé par un écrivain qui, en tant que tel, établissait une hiérarchie entre traduction et création, mais croyait aussi que la première constituait une phase préparatoire à la seconde. Cette conception était évidemment celle qui avait cours à la Renaissance, période historique qui a retrouvé les grands textes classiques dans leur pureté et veut les lire dans la langue vernaculaire. La traduction s'y pratique donc avec autant de fréquence que de ferveur. Les lettrés, comme Montaigne lui-même, ou encore comme Du Bellay, tenant d'une imitation qui, précisément, est une «innutrition» dans les classiques, sont nourris de ces textes de l'Antiquité - qu'ils ont toujours lus, et parfois traduits. Cependant, pour l'écrivain, il est nécessaire de dépasser le stade de la lecture et de la traduction pour atteindre celui de la création d'œuvres originales, création qui seule importe pour la littérature. Montaigne ne parlait donc que d'un point de vue d'écrivain. L'auteur veut transmettre à ses lecteurs un discours qui lui est propre. Il est convaincu que sa parole est originale et qu'elle rend compte d'un monde qui n'a pas encore été dit. Le traducteur, au contraire, n'a pas à parler de sa propre voix. Il n'a rien à dire que n'ait déjà dit un autre. Sa seule tâche est de permettre l'accès à un discours fidèlement «reproduit». José María Valverde, dans ce numéro même, insiste sur ce dernier aspect:

La traduction est une activité imitative. C'est comme le travail que réalisent les auteurs comiques qui imitent les hommes politiques et tentent de reproduire leur voix. (Traduire) c'est renoncer à soi. (C')est une activité qui a une grande valeur morale parce que c'est un exercice ascétique [...]; il faut s'oublier soi-même pour traduire. Si le traducteur a un style littéraire propre, il doit l'oublier.

L'activité du créateur et celle du traducteur ne sont donc pas complémentaires. Simplement elles se situent sur des plans différents. L'exercice de la traduction n'a rien de commun avec la création littéraire, pas plus qu'elle n'en a avec la théorie de la traduction. Tel serait peut-être le sens du manque d'intérêt des praticiens de la traduction en Espagne, quand il s'agit de se raconter eux-mêmes et de proposer une réflexion sur leurs «écrits».

C'est pourquoi, les auteurs dont les textes paraissent dans ce numéro font partie d'une minorité qui a consenti à prendre la parole. Par conséquent, ils se différencient $a$ priori des traducteurs qui n'ont pas désiré faire entendre leur voix, alors que, pour une fois, ils pouvaient cesser de parler par personne interposée. Il est évidemment significatif que ceux qui constituent l'exception - parce qu'ils ont accepté l'invitation à s'exprimer - soient tous écrivains et traducteurs à la fois, ou professeurs à l'université, traducteurs à leurs heures. Certains réunissent même la triple condition d'écrivain, de professeur et de traducteur. Aucun d'entre eux ne pratique donc la traduction comme une activité exclusive. S'il en est ainsi, c'est peut-être parce le traducteur-écrivain a, plus que les autres, l'habitude de tourner vers lui-même son propre regard, parce qu'il a intérêt à se situer par rapport à sa double activité, voire qu'il en a besoin. Sans doute a-t-il éprouvé aussi le plaisir que l'on prête à toute écriture autobiographique, même fragmentaire. Le traducteurprofesseur quant à lui, a l'obligation professionnelle de théoriser et de généraliser tout ce qui a rapport à la parole écrite et à ses processus de production, que le discours étudié soit celui de l'écrivain ou celui du traducteur. C'est donc, pour lui, une activité qui s'inscrit tout naturellement dans sa tâche quotidienne. Il n'est pas surprenant que ce type de traducteurs soient ceux qui aient accepté d'entreprendre «le projet de se peindre» qui leur était proposé.

Les traducteurs professionnels et ceux qui s'expriment dans les pages de ce numéro sont loin d'être dans la même situation par rapport à la traduction.

La première différence concerne le rapport de l'homme et du texte à traduire. Il est à supposer que la personne dont le métier est exclusivement celui de traducteur n'exerce pas un choix entre les textes sur lesquels elle peut/doit travailler. Par conséquent, elle n'éprouve 
pas, à leur égard, le désir de les affronter, de s'y mesurer. Elle n'a pas forcément d'affinités avec leur auteur. Son travail ne supposera des implications ni intellectuelles ni affectives et l'enjeu consistera uniquement à accomplir sa tâche avec compétence.

En revanche, on constatera qu'une des constantes chez les auteurs dont les textes sont reproduits ici, est d'avoir fait assez souvent des traductions qui n'était pas destinées à la publication (du moins immédiatement). Pour eux, c'est souvent le cas en début de carrière, la traduction est liée à une attirance à l'endroit d'un texte donné. Ces gens ont voulu traduire ce qu'ils avaient envie de lire, ce dont ils avaient envie de se pénétrer. C'est ce que dit Joaquim Mallafrè quand il donne la raison pour laquelle il a commencé à traduire :

I probably started translating because I did not know enough English to read Ulysses "directly».

Pour José María Valverde, il en va de même. Il s'est lancé dans l'entreprise de la traduction, comme poussé par une nécessité intellectuelle:

À dix-sept ans j'ai découvert Rilke et je me suis aperçu que ses vers devaient être lus en allemand, mais en même temps je devais les traduire pour les posséder complètement.

La traduction est, dans ce cas, une lecture menée à son point de perfection, qui transforme le texte étranger en un texte propre, dans un processus d'assimilation totale, de la part du lecteur-traducteur, d'une substance qui, ultérieurement, est à la fois sienne et autre. L'exercice traductif devient par là même une expérience d'ordre esthétique, sans aucun doute, d'importance vitale pour l'écrivain.

Tous les traducteurs-écrivains sont unanimes à reconnaître l'importance du texte traduit sur le créateur, au point où la frontière séparant l'œuvre créée et l'œuvre traduite — c'est-à-dire l'œuvre recréée - est totalement abolie par certains d'entre eux, quand ils s'arrêtent à considérer l'ensemble de leur production (Jaume Pérez Muntaner) :

Je n'ai aucun inconvénient à avouer que je considère mes traductions de poésie comme une continuation de mon œuvre de poète ni à dire que parfois elles ont (ou ont eu) une influence sur ma conception de l'œuvre littéraire [...] Bref je m'exprimerai d'une manière encore plus nette : ce sont aussi mes «créations» et je peux les placer sur le même plan que le reste de mes poèmes.

Aucun poète, aucun écrivain ne nie l'existence de réseaux d'influences - parfois subtiles, et qui ne tiennent, dans certains cas, qu'à une voix qui a emprunté son souffle à un auteur lointain - qui unit cultures, époques et écoles, par l'intermédiaire de la traduction. José María Valverde donne aussi l'exemple de l'influence de Hölderlin en Espagne et de son cheminement - qui paraîtrait plutôt fortuit - à travers les premières versions espagnoles de son œuvre:

Luis Cernuda avait un ami hispaniste dénommé Gebser qui avait écrit un livre sur Rilke en Espagne. Cet homme aida Cernuda à lire Hölderlin et lui traduisit même quelques textes. Comme conséquence, nous avons Invocaciones a la gracia del Mundo et Las nubes qui à leur tour ont permis l'existence, entre autres choses, de Sombra del Paraíso de Vicente Aleixandre.

L'implication affective du traducteur-écrivain dans les textes qu'il traduit, cette relation intime qu'il entretient avec eux, a parfois des conséquences assez inattendues, que seul le traducteur connaît vraiment. Ainsi, lorsqu'il se trouve en face d'cuvres qui n'ont pas répondu à son attente et qui l'ont déçu, le traducteur peut concevoir du ressentiment. Goethe a trompé José María Valverde et, en amoureux dépité, celui-ci s'est vengé. Il avoue, satisfait: «J'ai traduit (Goethe) sans pitié.» 
Il est très probable que ce ressentiment n'est pas l'apanage des seuls traducteursécrivains et que tout traducteur peut arriver à éprouver un sentiment négatif, en face d'une œuvre qui lui déplaît, lorsqu'il doit la transformer en quelque chose qui portera son nom (même si ce dernier figure en lettres minuscules...). Cependant le traducteur qui n'a pas choisi son texte, pour qui celui-ci n'a pas été objet de désir, le considère avec un détachement incompatible avec le sentiment de déception que nous venons d'évoquer.

Je m'attarderai sur un dernier fait qui concerne l'implication affective entre le traducteur et son texte, laquelle apparaît dans ces pages écrites par des traducteursécrivains-professeurs. Si le texte de départ est objet de désir, le texte d'arrivée, dans l'expérience de deux d'entre eux, l'est aussi très fortement, à cause de la langue choisie pour la traduction. Joaquim Mallafrè et Jaume Pérez Muntaner traduisent en catalan, langue minoritaire en Espagne et qui, durant de longues années de notre histoire encore récente, s'est vu refuser le statut de langue de culture. Si l'acte de traduction suscite chez ces deux traducteurs - Joaquim Mallafrè note cet aspect dans le texte qu'il publie ici un sentiment de fierté en raison de l'«honneur» linguistique retrouvé, l'expression, dans cette langue qui est profondément la leur, est avant tout la source d'un plaisir profond, de nature intellectuelle mais aussi affective, en étroite relation avec ce que chacun a de plus spécifique dans son expérience d'homme. Le traducteur recompose un texte étranger, le fond dans le moule de sa propre expression, en retrouvant les mots qui sont à la fois les plus lointains et les plus proches de son expérience dont sa langue maternelle, en l'occurrence, le catalan, a rendu compte au premier chef.

Il est néanmoins un point sur lequel l'essentiel du discours tenu par certains des auteurs de ce numéro est en accord avec ce que le silence évoqué auparavant laissait supposer. Plusieurs auteurs reprennent l'idée selon laquelle traduire suppose un savoir-faire dont l'acquisition se fait par «entrainement», avec l'obligation, pour le traducteur, de résoudre phrase après phrase, page après page, des difficultés toujours différentes, d'assumer des choix toujours personnels. C'est ce que José María Valverde, dès l'abord, exprime de manière concise et définitive :

Mon sujet ne peut être la théorie de la traduction. Je n'ai pas de théorie et, qui plus est, je ne crois pas en la théorie de la traduction.

Ce rejet a priori de ce qui serait réflexion théorique s'inspirant d'une pratique ou s'inscrivant dans ses marges peut surprendre de la part d'un traducteur. Serait-il concevable, par exemple, de refuser à la langue la possibilité d'être un objet d'étude, sous prétexte que parler ou écrire dans une langue relève de la pratique? Pour qui s'occupe de traduction, la situation a donc un côté tout à fait paradoxal qu'on pourrait résumer de la manière suivante: certains traducteurs traduisent, mais refusent la légitimité du discours sur la traduction et, du coup, l'existence de la traductologie.

Il est évident qu'il existe, sinon une théorie, du moins une théorisation de la traduction. En 1993, nous sommes en présence, d'une masse importante de textes ${ }^{1}$ qui analysent la traduction et la constituent en objet d'une discipline appartenant aux sciences du langage. Le numéro suivant contient quelques réflexions de ce genre, par exemple, celles de Jenaro Talens qui montrent que la traduction n'est pas «transvasement» de sens d'une langue à une autre, mais «re-création», lieu d'une écriture productrice de sens nouveaux.

Un des arguments auquel les traducteurs recourent parfois pour refuser une légitimité à la recherche en traductologie est que ses responsables ne sont généralement pas eux-mêmes des traducteurs, c'est-à-dire que l'objet de leur étude leur est «extérieur». Cependant, il est évident que ce fait ne constitue aucun obstacle en principe à la réflexion théorique. En schématisant les choses à l'extrême, on peut dire qu'il est possible, par 
exemple, de décrire l'organisation sociale des Indiens de l'Amazonie sans être soi-même un Indien. En traductologie, il en va de même.

Il est évident, aussi, que les perspectives adoptées pour la théorisation sur la traduction (méthode, cadre, théorie linguistique sous-jacente) sont très différentes. Cependant, cet argument n'est pas davantage un argument recevable: la diversité est la condition du progrès scientifique et l'hétérogénéité des fins ne peut invalider le résultat des recherches dans ce domaine.

En tout état de cause, en ce qui concerne le refus de théorisation de certains des traducteurs espagnols qui ont pris la parole ici, et plus largement, en ce qui concerne l'Espagne et sa situation spécifique dans le domaine de la traduction, il convient tout d'abord de se demander si la tendance que nous venons d'évoquer dans notre cadre restreint (et qui s'est fait jour dans quelques-unes de ces pages), peut être significative de l'ensemble des positions des traducteurs de ce pays : ce clivage entre praticiens (refusant la théorisation) et théoriciens existe-t-il véritablement? La question vaut-elle la peine d'être posée?

Quoi qu'il en soit, si nous voulons répondre à la question, il nous faudra tenir compte, obligatoirement, $\mathrm{du}$ fait que les études théoriques sur la traduction sont encore très récentes en Espagne et qu'elles n'ont pas encore beaucoup de lieux où s'exprimer. Quelques chaires de littérature sont (ont été) occupées par de brillants traducteurs qui s'intéress(ai)ent peu à la traductologie, alors que, du côté de la théorie, seules quelques rares écoles de traducteurs et d'interprètes rattachées à des universités ont mis sur pied des publications périodiques (et pas toujours de façon régulière...), tandis que quelques départements universitaires de langues vivantes ont parfois osé faire des incursions dans cette terra nullius qui n'avait pas droit de cité dans les facultés.

Cependant, il semble qu'à l'heure actuelle l'université espagnole se soit décidée à reconnaître l'existence de la traduction dans les facultés. Le diplôme de Licencié en traduction et en interpétation vient d'être créé, ce qui entraînera la mise sur pied, sinon de départements de traduction, du moins d'unités d'enseignement et de recherche pourvus d'une certaine homogénéité. L'institution universitaire offre ainsi un cadre à la recherche en traductologie. À celle-ci de trouver le moyen de se développer, les prérequis ayant été assurés.

Je terminerai en soulignant que, si du point de vue de l'histoire de la traduction en Espagne, ce numéro recueille des données et présente des perspectives dont il faudra tenir compte, il présente aussi un intérêt indéniable sous d'autres aspects. Certains de ces articles sont des textes d'un intérêt indubitable du point de vue de l'histoire intellectuelle espagnole telle qu'elle apparaît en filigrane dans ces pages. C'est le renouveau de l'édition espagnole de l'après-guerre, la renaissance du catholicisme observée dans les années 60 et les traductions auxquelles elle donnera lieu, la lutte pour une université indépendante - et plus généralement contre la dictature de Franco - laquelle signifie l'exil de certains universitaires de l'époque.

Les coups d'œil rétrospectifs de quelques-uns des auteurs de ces pages, dont deux d'entre eux furent des acteurs importants dans cette histoire, nous font regretter très profondément que ces derniers ne prennent pas la plume pour se situer personnellement par rapport à leur époque et à leurs travaux dans un projet autobiographique plus vaste.

Cependant tels qu'ils se présentent, ces «lambeaux de pourpre» autobiographiques ont aussi une valeur littéraire. Ils retracent des itinéraires professionnels, mais rendent compte aussi d'événements plus personnels liés à des expériences esthétiques essentielles dans la vie des auteurs-traducteurs. De ce point de vue, nous regrettons l'absence de tradition où les maîtres d'une génération pourraient se raconter et nous faire partager quelque peu, rétrospectivement, leur vie. 
En ce qui me concerne, il me reste à espérer d'avoir traduit adéquatement les textes de traducteurs sur la traduction. Tel, du moins, était mon désir. Cependant, le passage à l'acte, en traduction (matière qui, on l'a vu, n'est pas étrangère à la création), implique inévitablement un sentiment de déception: Valéry disait à juste titre, que l'œuvre réalisée est toujours moins belle que l'œuvre rêvée. La première, cependant, a le mérite d'exister...

\section{Note}

1. Ces textes sont essentiellement français, anglais, allemands et russes. Les Espagnols ne participent que depuis peu à cette production. Les grands textes espagnols de notre époque sur la traduction, par exemple celui de J. Ortega y Gasset ( Miseria y esplendor de la Traducción (1937) ou ceux de V., sont, comme le signale J.-R. Ladmiral, «d'obédience philosophique» (Jean-René Ladmiral, 1987, «Traductologiques», numéro spécial Le Français dans le monde, «Retour à la traduction» p. 20). Valentín García Yebra, autre grand nom de la traduction espagnole dont le texte figure dans ce numéro, réfute le terme «traductologie». 